\title{
A Case of Organizing Pneumonia (OP) Associated with Pembrolizumab
}

\section{Paraskevi Fragkou1, Maria Souli ${ }^{1}$, Maria Theochari², Christina Kontopoulou ${ }^{3}$, Stelios Loukides ${ }^{4}$ and Anna Koumarianou ${ }^{2}$}

${ }^{1}$ Fourth Department of Internal Medicine, Infectious Diseases Unit, Attikon University Hospital, National and Kapodistrian University of Athens, Athens, Greece. ${ }^{2}$ Fourth Department of Internal Medicine, Hematology-Oncology Unit, Attikon University Hospital, National and Kapodistrian University of Athens, Athens, Greece. ${ }^{3} 2$ nd Radiology Department, Attikon University Hospital, National and Kapodistrian University of Athens, Athens, Greece. ${ }^{4}$ 2nd Respiratory Medicine Department, Attikon University Hospital, National and Kapodistrian University of Athens, Athens, Greece.

\begin{abstract}
Until recently, chemotherapy for metastatic melanoma had disappointing results. The identification of immune checkpoints such as CTLA-4 and PD-1/PD-L1 has led to the development of an array of monoclonal antibodies (Mabs). These immunologic approaches against tumoral cells come with a novel kind of side effects that the clinician needs to be familiarized with. Herein, we report for the first time a case of organizing pneumonia, based on imaging and cytological analyses of bronchoalveolar lavage, possibly associated with the use of pembrolizumab, an anti-PD-1 Mab recently approved for the treatment of metastatic melanoma.
\end{abstract}

KEYWORDS: OP, organizing pneumonia, pembrolizumab, melanoma, side effect

CITATION: Fragkou et al. A Case of Organizing Pneumonia (OP) Associated with Pembrolizumab. Drug Target Insights 2016:10 9-12 doi:10.4137/DTI.S31565.

TYPE: Case Report

RECEIVED: February 5, 2016. RESUBMITTED: April 14, 2016. ACCEPTED FOR PUBLICATION: April 18, 2016.

ACADEMIC EDITOR: Anuj Chauhan, Editor in Chief

PEER REVIEW: Three peer reviewers contributed to the peer review report. Reviewers' reports totaled 454 words, excluding any confidential comments to the academic editor. FUNDING: Authors disclose no external funding sources.

COMPETING INTERESTS: Authors disclose no potential conflicts of interest.

COPYRIGHT: ๑) the authors, publisher and licensee Libertas Academica Limited. This is an open-access article distributed under the terms of the Creative Commons CC-BY-NC 3.0 License.

\section{CORRESPONDENCE: akoumari@yahoo.com}

Paper subject to independent expert single-blind peer review. All editorial decisions made by independent academic editor. Upon submission manuscript was subject to anti-plagiarism scanning. Prior to publication all authors have given signed confirmation of agreement to article publication and compliance with all applicable ethical and legal requirements, including the accuracy of author and contributor information, disclosure of competing interests and funding sources, compliance with ethical requirements of competing interests and funding sources, compliance with ethical requirements
relating to human and animal study participants, and compliance with any copyright relating to human and animal study participants, and compliance with any copyright
requirements of third parties. This journal is a member of the Committee on Publication Ethics (COPE)

Provenance: the authors were invited to submit this paper.

Published by Libertas Academica. Learn more about this journal.

\section{Introduction}

New monoclonal antibodies (Mabs) targeting the immune checkpoint blockade have revolutionized the treatment of advanced melanoma patients. Although these novel Mab therapies can induce remarkable antitumor-specific immune responses, they have distinct side effects as compared with chemotherapy, including nonspecific immunological activation. ${ }^{1}$ To our knowledge, we report the first case of organizing pneumonia (OP) in a melanoma patient under immunotherapy with pembrolizumab, a PD-1 targeting Mab. The patient's next of kin has given consent for publication of this report.

\section{Case Presentation}

A 64-year-old woman presented to our emergency department with a 10-day history of progressive dyspnea and dry cough nonresolving after a 9-day course of oral antibiotics (levofloxacin $750 \mathrm{mg}$ daily). She had been diagnosed with a c-kit/BRAF wild-type, stage IV mucosal melanoma of the nasal cavity with involvement of cervical and mediastinal lymph nodes and metastases to the liver, brain, and bones nine months earlier. Initial treatment included two cycles of dacarbazine $1000 \mathrm{mg} / \mathrm{m}^{2}$ and zoledronic acid $4 \mathrm{mg}$ every three weeks. One month later, the patient experienced disease progression and was treated with four cycles of ipilimumab immunotherapy $3 \mathrm{mg} / \mathrm{kg}$ and zoledronic acid $4 \mathrm{mg}$ q3 weeks intravenously (iv). Three months later, the disease advanced further, and a second-line immunotherapeutic agent with pembrolizumab was initiated. Thirteen days prior to her admission, she had received her fourth cycle of immunotherapy with pembrolizumab. Her pretherapeutic thoracic CT scan was normal.

On examination, she was pale and tachypneic. The chest auscultation revealed diffuse bilateral coarse crackles and areas of bronchial breathing mainly in the right middle lobe. She was afebrile, but her oxygen saturation on room air was $75 \%$.

Her blood tests were normal except an erythrocyte sedimentation rate of $200 \mathrm{~mm} /$ hour, a white blood count of 10,970 cells/ $\mu \mathrm{L}$ (differential: $83.6 \%$ neutrophils, 9.7\% lymphocytes, and $6.7 \%$ monocytes), and a C-reactive protein of $173 \mathrm{mg} / \mathrm{L}$ (normal value: 0-6 mg/L). A chest radiography demonstrated patchy alveolar infiltrates mainly on the right upper, the right middle, and the left upper lobe.

An initial diagnosis of a lower respiratory tract infection was made, and antibiotics were commenced, including ceftriaxone $2 \mathrm{~g} /$ day iv and azithromycin $500 \mathrm{mg} /$ day orally. After three days of treatment, there was no improvement of signs or symptoms. Subsequently, the patient underwent a high-resolution computed tomography (HRCT) of the chest and fiberoptic bronchoscopy with bronchoalveolar lavage (BAL). Samples were submitted for staining and culture for 


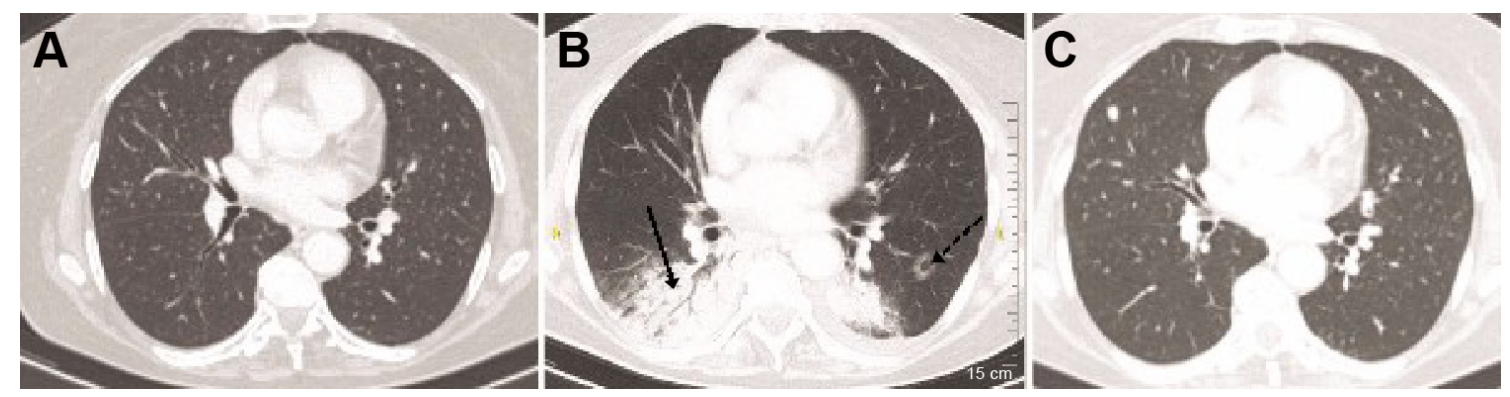

Figure 1. Patient's computed tomography of chest indicating (A) baseline CT scan before pembrolizumab therapy, (B) reverse halo sign (dotted arrow) and consolidation with air bronchogram (straight arrow) features of OP following therapy of pembrolizumab, and (C) follow-up CT scan indicating complete resolution of the organized pneumonia, one month after the steroid therapy. Metastatic lesion in the right middle lobe.

bacteria, fungi, mycobacteria, and nocardia, special staining for Pneumocystis jirovecii, cytology, immunophenotyping, and molecular testing for viral infection. The HRCT demonstrated patchy consolidation with a predominantly subpleural and peribronchial distribution, small ill-defined peribronchiolar nodules, tree-in-bud sign, and reverse halo sign, involving all lobes mainly the lower ones (Fig. 1). There was no lymph nodal enlargement or pleural effusion. Cytology, microbiology, and molecular testing were negative for malignant or infectious diseases. The immunophenotyping of the BAL lymphocytes by flow cytometry revealed macrophage and monocyte predominance $(60.7 \%)$ with a lymphocyte count of $28.7 \%$ and a $\mathrm{CD} 4+/ \mathrm{CD} 8+$ index of 0.4 . Based on the imaging and clinical and BAL findings that were consistent with OP, therapy with corticosteroids (prednisolone $50 \mathrm{mg} /$ day iv) was initiated that resulted into rapid clinical and radiographic improvement of the patient. Her C-reactive protein declined to $20 \mathrm{mg} / \mathrm{L}$ within two days of steroid treatment. The treating physician assessed that OP represented a grade 3 adverse event, ${ }^{2}$ most probably related to treatment with pembrolizumab, which was discontinued. Three months later, the patient died of advanced metastatic disease in the brain.

\section{Discussion}

Advanced melanoma has been a challenge in oncology as the five-year survival rates were immensely low with standard chemotherapy. ${ }^{3}$ Newer Mab therapies targeting the immune checkpoint, ipilimumab (Yervoy; Bristol-Myers Squibb), nivolumab (Opdivo; Bristol-Myers Squibb), and pembrolizumab (Keytruda; Merck Sharp \& Dohme Corp) were shown to prolong progression free and overall survival compared with chemotherapy. ${ }^{4}$

Pembrolizumab is a humanized IgG4 Mab raised against the programmed cell death protein 1 (PD-1) immune checkpoint, recently approved by the US Food and Drug Administration and the European Medicines Agency for the treatment of advanced melanoma, as a result of a randomized controlled phase III trial showing improved overall survival. ${ }^{5}$

PD-1 is a cell surface receptor that belongs to the immunoglobulin superfamily and is normally expressed on B-cells, ${ }^{6}$
T-cells, ${ }^{7}$ and macrophages. ${ }^{8}$ PD-1 and its ligands, namely, programmed cell death ligand 1 (PD-L1) and 2 (PD-L2), have been found to be abnormally expressed by tumor cells. ${ }^{9}$ These molecules play an important role in inhibiting antitumor immune responses and are responsible for the immune evasion of cancer cells by the cytotoxic effector immune cells. Mab therapies against the PD- 1 can reverse immune tolerance and achieve high tumor response rates and improvement of survival. ${ }^{10}$

The therapeutic effects of Mabs are not fully understood, but they are mediated through direct signaling, complementdependent cellular cytotoxicity, and antibody-dependent cellular cytotoxicity. ${ }^{11}$

Pemprolizumab (previously known as MK-3475 or lambrolizumab) is a humanized monoclonal IgG4-kappa isotype antibody. Its variable region sequences were derived from a very high-affinity mouse antihuman PD-1 antibody (dissociation constant, $28 \mathrm{pM}$ ) that was grafted into a human IgG4 immunoglobulin with a stabilizing S228P Fc alteration that does not engage $F_{c}$ receptors or activate complement, thus avoiding cytotoxic effects of the antibody when it binds to the PD-1 receptor of T-cells that are intended to activate against tumor cells. ${ }^{12}$ It is reasonable to assume that the mechanism of action and the resulting side effects of pembrolizumab are mediated through PD-1 direct signaling on T-cells or macrophages.

Cryptogenic organizing pneumonia (COP), the idiopathic form of OP (formerly called bronchiolitis obliterans $\mathrm{OP}$ ), is a type of diffuse interstitial lung disease that affects the distal bronchioles, respiratory bronchioles, alveolar ducts, and alveolar walls. The primary area of injury is within the alveolar wall. Since many cases of COP are secondary, it is more proper to use the term $\mathrm{OP}$ associated with the name of the secondary cause. ${ }^{13}$

Histopathologic study through lung biopsy via videoassisted thoracoscopic surgery or open thoracotomy is the optimal method for establishing the diagnosis of COP, in order to have enough tissue for the pathologist to exclude other processes. Because of the low oxygen saturation level, we selected to combine the cytological profile of BAL with 
characteristic clinical and imaging features. A previous study has already described in detail the cytological and immunocytological profile of BAL in COP, which include foamy macrophages, mast cells, plasma cells, a decreased CD4/CD8 T-cell ratio, an increase in activated T-lymphocytes based on HLA-DR or interleukin-2 receptor expression and a lymphocyte increase of $20 \%-40 \%$ also documented in our patient with bronchiolitis obliterans.

The most commonly occurring clinical findings in $\mathrm{COP}$ are similar to our patient's symptoms, including nonproductive persistent cough, dyspnea, and malaise without fever. ${ }^{14}$ Since the most common manifestations are nonspecific, diagnosis is often delayed (6-13 weeks), and similar to our patient, a lack of response to empiric antibiotics for community-acquired pneumonia may be the initial clue to the presence of a noninfectious, inflammatory pneumonia. ${ }^{15}$ Similar to that in our patient, HRCT has findings that are characteristic for COP and include peripheral bilateral patchy opacities, more frequently in the periphery and in the lower lung zone, air bronchogram, and reverse halo sign..$^{16}$ Once the diagnosis of COP is made, rapid clinical and imaging improvement is obtained with corticosteroid treatment, but relapses are common after stopping treatment, indicating an established immune reaction requiring long-term therapy. ${ }^{15}$

When COP is suspected in cancer patients, differential diagnoses that need to be considered include disease progression, cardiogenic edema, radiation pneumonitis, allergies, pulmonary hemorrhage, and infections that cause diffuse interstitial infiltrates in the lungs such as gram-negative or grampositive bacteria, fungi (Aspergillus, Candida, and P. jirovecii), parasites (Toxoplasma gondii), or viruses (herpes simplex, varicella zoster, and cytomegalovirus). ${ }^{17}$

On the other hand, pneumonitis in cancer patients may hold a heterogeneous pathological background based on various antineoplastic agents including chemotherapy, such as taxanes, targeted therapy, such as everolimus, and Mabs, such as rituximab, a chimeric $\operatorname{IgG} 1{ }^{17}$

In retrospective analysis of patients with non-Hodgkin lymphoma treated with rituximab, 8 of 23 patients, diagnosed with COP and treated with corticosteroids, died, indicating that $\mathrm{COP}$ although rare may become a fatal pulmonary toxicity and the oncologists or internists must maintain a high index of suspicion to recognize this complication early.

Little is known regarding the clinicopathologic features of pneumonitis related to the novel immune checkpoint inhibitors. A recent study indicated the development of pneumonitis in three patients treated with nivolumab, an anti-PD-1 Mab, 7-24.3 months after treatment initiation. ${ }^{18}$ Two patients were radiographically found with acute interstitial pneumonia/acute respiratory distress syndrome, and one patient was with nonspecific interstitial pneumonia. Two of these patients required admission in the intensive care unit, and one succumbed four weeks after diagnosis of pneumonitis. Computed tomography findings differed from $\mathrm{COP}$, and BAL or transbronchial biopsy was not performed in these patients. Another report involving a patient treated with nivolumab described an OP diagnosed both radiographically and pathologically. ${ }^{19}$ Similar to our case, CT of the chest showed the typical reversed halo sign and had a favorable response to corticosteroid therapy.

With novel immunotherapies, the incidence of autoimmune phenomena is rising; therefore, it is important to recognize an OP early in any patient receiving anti-PD-1 and presenting with symptoms of new cough or shortness of breath. The diagnosis needs to be supported by imaging and cytological investigations. Although the exact causative mechanism of OP by anti-PD-1 Mab therapy cannot be established as yet, a T-cell or macrophage-driven effect is the most plausible explanation, and further studies are warranted to define the pathogenic mechanism underlying this possibly lethal side effect.

\section{Acknowledgment}

The authors are obliged to Professor Dimitrios Boumpas for critical revision of the manuscript.

\section{Author Contributions}

Conceived and designed the experiments: AK. Analyzed the data: MS. Wrote the first draft of the manuscript: PF. Contributed to the writing of the manuscript: $\mathrm{PF}, \mathrm{MS}, \mathrm{MT}, \mathrm{CK}$, SL, AK. Agree with manuscript results and conclusions: PF, MS, MT, CK, SL, AK. Jointly developed the structure and arguments for the paper: PF, MS, MT, CK, SL, AK. Made critical revisions and approved final version: PF, MS, MT, CK, SL, AK. All authors reviewed and approved of the final manuscript.

\section{REFERENCES}

1. Postow MA, Callahan MK, Wolchok JD. Immune checkpoint blockade in cancer therapy. J Clin Oncol. 2015;33(17):1974-1982.

2. National Cancer Institute, National Institutes of Health, U.S. Department of Health and Human Services. Common Terminology Criteria for Adverse Events (CTCAE). Version 4.0. Bethesda, MD: NIH. Published: May 28, 2009 (v4.03: June 14, 2010). NIH Publication No. 09-5410. Revised June 2010.

3. McDermott D, Lebbe C, Hodi FS, et al. Durable benefit and the potential for long-term survival with immunotherapy in advanced melanoma. Cancer Treat Rev. 2014;40:1056-1064.

4. Eggermont AM, Maio M, Robert C. Immune checkpoint inhibitors in melanoma provide the cornerstones for curative therapies. Semin Oncol. 2015;42:429-435.

5. Robert C, Schachter J, Long GV, et al. Pembrolizumab versus ipilimumab in advanced melanoma. N Engl J Med. 2015;372:2521-2532.

6. Thibult ML, Mamessier E, Gertner-Dardenne J, et al. PD-1 is a novel regulator of human B-cell activation. Int Immunol. 2013;25:129-137.

7. Jin HT, Ahmed R, Okazaki T. Role of PD-1 in regulating T-cell immunity. Curr Top Microbiol Immunol. 2011;350:17-37.

8. Huang X, Venet F, Wang YL, et al. PD-1 expression by macrophages plays a pathologic role in altering microbial clearance and the innate inflammatory response to sepsis. Proc Natl Acad Sci U S A. 2009;106:6303-6308.

9. Wu P, Wu D, Li L, Chai Y, Huang J. PD-L1 and survival in solid tumors: a meta-analysis. PLoS One. 2015;10:e0131403.

10. Pardoll DM. The blockade of immune checkpoints in cancer immunotherapy. Nat Rev Cancer. 2012;12:252-264.

11. Weiner GJ. Rituximab: mechanism of action. Semin Hematol. 2010;47:115-123. 
12. Hamid O, Robert C, Daud A, et al. Safety and tumor responses with lambrolizumab (anti-PD-1) in melanoma. N Engl J Med. 2013;369:134-144.

13. Travis WD, Costabel U, Hansell DM, et al. An official American Thoracic Society/European Respiratory Society statement: update of the international multidisciplinary classification of the idiopathic interstitial pneumonias. $\mathrm{Am}$ J Respir Crit Care Med. 2013;188:733-748.

14. Barker AF, Bergeron A, Rom WN, et al. Obliterative bronchiolitis. $N$ Engl JMed. 2014;370:1820-1828.

15. Cordier JF. Cryptogenic organising pneumonia. Eur Respir J. 2006;28:422-446.

16. Nishimura K, Itoh H. High-resolution computed tomographic features of bronchiolitis obliterans organizing pneumonia. Chest. 1992;102:26S-31S.
17. Kim KM, Kim HC, Jeon KN, et al. Rituximab-CHOP induced interstitial pneumonitis in patients with disseminated extranodal marginal zone B cell lymphoma. Yonsei Med J. 2008;49:155-158.

18. Nishino M, Sholl LM, Hodi FS, et al. Anti-PD-1-related pneumonitis during cancer immunotherapy. NEngl J Med. 2015;373:288-290.

19. Nakashima K, Naito $T$, Omori $S$, et al. Organizing pneumonia induced by nivolumab in a patient with metastatic melanoma. J Thorac Oncol. 2016;11(3): $432-433$. 